\title{
Use of DAMPs and SAMPs as Therapeutic Targets or Therapeutics: A Note of Caution
}

\author{
Walter Gottlieb Land ${ }^{1,2}$ (1)
}

Published online: 4 April 2020

(c) Springer Nature Switzerland AG 2020

\begin{abstract}
This opinion article discusses the increasing attention paid to the role of activating damage-associated molecular patterns (DAMPs) in initiation of inflammatory diseases and suppressing/inhibiting DAMPs (SAMPs) in resolution of inflammatory diseases and, consequently, to the future roles of these novel biomarkers as therapeutic targets and therapeutics. Since controlled production of DAMPs and SAMPs is needed to achieve full homeostatic restoration and repair from tissue injury, only their pathological, not their homeostatic, concentrations should be therapeutically tackled. Therefore, distinct caveats are proposed regarding choosing DAMPs and SAMPs for therapeutic purposes. For example, we discuss the need to a priori identify and define a context-dependent "homeostatic DAMP:SAMP ratio" in each case and a "homeostatic window" of DAMP and SAMP concentrations to guarantee a safe treatment modality to patients. Finally, a few clinical examples of how DAMPs and SAMPs might be used as therapeutic targets or therapeutics in the future are discussed, including inhibition of DAMPs in hyperinflammatory processes (e.g., systemic inflammatory response syndrome, as currently observed in Covid-19), administration of SAMPs in chronic inflammatory diseases, inhibition of SAMPs in hyperresolving processes (e.g., compensatory antiinflammatory response syndrome), and administration/induction of DAMPs in vaccination procedures and anti-cancer therapy.
\end{abstract}

\section{Introduction}

Twenty-six years ago, the danger/injury model in immunology was formulated and published, holding that the immune system does not care about "self" and "non-self," but that it is any form of cell stress/tissue injury, rather than the presence of a non-self (i.e., genetically foreign entitied) that induces immunity [1,2]. The core of the danger/injury model refers to the generation of damage-associated molecular patterns (DAMPs), a term coined by Land [3], also denoted as "danger signals" in the international literature. Current notions hold that all organisms on our planet use DAMPs for their defense against any cell stress and tissue injury [4]. Indeed, the evolutionarily determined function of DAMPs is to promote injury-induced defense responses, the aim always being to repair damaged tissue - that is, to

Walter Gottlieb Land

land.w.damps@gmail.com

1 Molecular ImmunoRheumatology, INSERM UMR_S1109, Laboratory of Excellence Transplantex, University of Strasbourg, Strasbourg, France

2 German Academy for Transplantation Medicine, Munich, Germany restore homeostasis. Unique germline-encoded endogenous molecules accomplish this goal by promoting contextdependent proinflammatory responses, which transition into inflammation-resolving pathways that are driven by suppressing/inhibiting DAMPs (SAMPs), a term coined by Land $[5,6]$ for molecules counteracting DAMPs. Moreover, in the presence of altered-self or non-self antigens, the DAMPs shape adaptive immune responses that have the same homeostasis-restoring aim.

However, there is another side of the coin: emerging evidence from preclinical and clinical studies suggests that DAMPs and SAMPs play a critical role in the pathogenesis of human diseases. For example, excessive or prolonged production of DAMPs and/or impaired generation of SAMPs may promote hyperinflammation or chronic inflammation. Consequently, these molecules are now considered valuable diagnostic and prognostic biomarkers in human disorders [5, 7-11]. Moreover, the pathogenetic involvement of these molecules qualifies them as relevant drug targets or therapeutics as well. Indeed, accumulating preclinical data have encouraged envisaging DAMPs as therapeutic targets for inhibition [5, 12-17] and administration of SAMPs as candidate drugs [5, 18-27] for treating acute or chronic inflammatory disorders. On the other hand, DAMPs may also be 


\section{Key Points}

Damage-associated molecular patterns (DAMPs) and their counteracting molecules, the suppressing/inhibiting DAMPs (SAMPs), are the core of the danger/injury model in immunology, which holds that any form of cell stress/ tissue injury (via controlled emission of DAMPs), rather than the presence of non-self (i.e., genetically foreign entities), initiates a protective immune defense response.

However, in the case of uncontrolled, dysregulated emission of DAMPs and SAMPs, these molecules are involved in the pathogenesis of many acute and chronic inflammatory diseases. This recent groundbreaking knowledge has stimulated researchers and clinicians to exploit these molecules as therapeutic targets or therapeutics.

Given the fact that controlled emission of DAMPs and SAMPs is vital for successful host defense and that it is critical for restoring homeostasis, their therapeutic inhibition in the case of pathological (excessive or permanent) emission has to be performed under strict caveats and precautions. Therefore a homeostatic window of DAMP and SAMP concentrations may be defined, and concentrations should not be outside the window's upper and lower bounds in the course of any treatment modality.

therapeutically administered when enhancement of adaptive immune responses, such as antitumor immune responses [28-32], are desired.

Collectively, both DAMPs and SAMPs appear highly suitable to serve as future therapeutic targets or therapeutics in daily clinical practice. Hence, focusing on some practical aspects, a few points regarding this emerging field in modern medicine are worth addressing in terms of an introduction to the future use of these molecules clinically.

\section{DAMP-Promoted Initiation of Inflammation and SAMP-Driven Resolution of Inflammation as Fundamental Defense Principles in Restoring Homeostasis upon Any Cell Stress and/or Tissue Injury}

\subsection{Introductory Remarks}

DAMP-promoted initiation of inflammation and SAMPdriven resolution of inflammation are responses to any infectious/sterile cell stress/tissue injury, that is, adaptive responses aiming to restore homeostasis [5]. The key adaptation strategy is to promote the restoration of homeostasis by returning the regulated variables (here DAMPs and SAMPs) to homeostatic set points. Another strategy is to switch the homeostatic set points to different values of DAMPs and SAMPs that are better suited for dealing with a given extreme hyperacute or chronic abnormal condition.

\subsection{DAMP-Promoted Initiation of Inflammation}

DAMPs such as high mobility group box 1 (HMGB1), S100 proteins, nuclear and mitochondrial DNA (nDNA and mtDNA), and extracellular histones are recognized by their cognate pattern recognition receptors (PRRs) on/ in cells of the innate immune system (e.g., leukocytes, M1 macrophages) and, following this process, drive inflammatory responses. As comprehensively reviewed [5], all possible tools of the innate immune system, involving PRR-triggered, proinflammatory cellular, humoral, and molecular pathways, can get activated by DAMPs, in the case of an infectious injury together with microbeassociated molecular patterns (MAMPs), also called pathogen-associated molecular patterns (PAMPs). Secretion by DAMP-activated cells of cytokines, chemokines, and adhesion molecules, associated with leukocyte recruitment/infiltration are among the most prominent features of this inflammatory scenario. An integral part of these DAMP-promoted responses is the process of phagocytosis, which can be regarded as a critical mechanism of innate immunity, and is usually accompanied by humoral innate effector responses, including activation of the complement system.

\subsection{SAMP-Driven Resolution of Inflammation}

SAMPs include molecules such as prostaglandin E2 (PGE2), annexin A1 (AnxA1), and specialized proresolving mediators (SPMs) (reviewed in [5]). Here, SAMPorchestrated inflammation-resolving responses are exemplified by the action of SPMs as the best-studied molecules so far. In fact, the distinct functions of SPMs are multifactorial. These functions include the ability to terminate infiltration of inflammatory leukocytes into injured tissue and to mediate stop signals to limit further leukocyte recruitment. Further, SPMs have been shown to enhance macrophage phagocytosis of apoptotic leukocytes (efferocytosis) and cellular debris. In turn, macrophages, during the process of efferocytosis, were observed to produce SPMs [33, 34]. Moreover, SPMs have been demonstrated to decrease excessive oxidative stress [35], to augment bacteria killing by phagocytes, to act at both the transcriptional and translational level, and to shorten time to resolution, via activation of endogenous 
resolution programs (see [36]). For example, resolvin E1 (RvE1) and RvE2 were found to stimulate the antiinflammatory cytokine interleukin 10 and phagocytosis potently. Moreover, lipoxin A4 (LXA4), RvD1, and RvE1, as well as the maresin $(\mathrm{MaR})$ intermediate eMaR, were observed to stimulate transdifferentiation of M1-like to M2-like macrophages committed to execute tissue repair and regeneration [37-42].

\section{DAMPs and SAMPs in Therapy: A Design of Guidelines}

\subsection{Introductory Remarks}

Plausibly, the controlled, context-dependent production of inflammation-initiating DAMPs and inflammation-resolving SAMPs, followed by subsequent DAMP-promoted tissue repair and regeneration, is needed to achieve full homeostatic restoration from tissue injury and, thus, must not be disturbed by therapeutic interventions.

In contrast, uncontrolled pathological production of DAMPs and/or SAMPs in the course of a disease may reflect their dysregulated (increased or decreased) emission upon infectious/sterile stress/injury. It is this indication where DAMPs or SAMPs can be exploited as therapeutic targets or therapeutics.

Hence, the routine use of DAMPs or SAMPs as therapeutic targets or therapeutics in patients is a new challenge for the medical profession and, therefore, requires a careful discussion of regulations, caveats, and adaptations. Some thoughts on this emerging hot topic in modern medicine are discussed in the following sections.

\subsection{Controlled Inflammatory Response Upon Injury: A Reference Point for Identification of a "Homeostatic DAMP:SAMP Ratio" and a "Homeostatic Window" of DAMP and SAMP Concentrations}

\subsubsection{General Remarks}

The new knowledge about a controlled, protective inflammatory response upon injury refers to a DAMP-driven proinflammatory crescendo $\rightarrow$ decrescendo that proceeds (slightly shifted, but nearly in parallel with) a SAMP-orchestrated proresolving reversed crescendo $\rightarrow$ decrescendo (see Fig. 1). As plausible and reasonable as this definition may be in light of recent research work in inflammation, it will be difficult to safely implement this new knowledge in clinical practice. Some thoughts about this challenge are expressed in the following sections.

\subsubsection{The "Homeostatic DAMP:SAMP Ratio"}

When considering DAMPs and SAMPs as therapeutic targets or their use as therapeutics, as well as for safety reasons, one may design and apply a chronologically occurring ratio of DAMPs and SAMPs as a reference point. The selected homeostatic set point would operate in the course of a given and defined controlled infectious/sterile injury-induced inflammatory response resulting in wound healing in terms of a restitutio ad integrum. Such a "homeostatic DAMP:SAMP ratio" may also be called a "homeostatic DAMP:SAMP signature," which is required to achieve successful wound healing upon injury without further complications. According to such a model, injury-induced DAMPs are dominating during the initial proinflammatory phase, reflecting a DAMP:SAMP ratio greater than $1(>1)$. Via an intermediate ratio of \pm 1 , the DAMP:SAMP ratio then inverses during the resolving phase to less than $1(<1)$, and the restitutio ad integrum is finally marked by "1" (the "homeostatic set point") (Fig. 1). This is complicated by the fact that usually several key DAMPs and SAMPs are acting in a given inflammatory response (which may be called a "homeostatic DAMP:SAMP pattern ratio"). Hence, in this situation, the course of the blood levels for each molecule during the inflammatory phase and the resolving phase must be determined to calculate the ratio.

\subsubsection{The "Homeostatic Window" of DAMP and SAMP Concentrations}

As suggested above, before any consideration is given to choosing DAMPs or SAMPs as therapeutic targets to be inhibited, the homeostatic concentrations of these molecules (for example, in body fluids) required to establish successful wound healing after injury (restitutio ad integrum) have to be a priori identified. The aim is to define a homeostatic DAMP:SAMP ratio in a given situation. More precisely, and for safety reasons, a "homeostatic window" for DAMP and SAMP concentrations might be worked out in orientating clinical trials aimed at guiding the chosen therapy (Fig. 1). The upper boundary of the homeostatic window for DAMPs should not be exceeded because of the increased risk of hyperinflammation and organ dysfunction, and the lower boundary of the window should also not be crossed because of the increased risk of compromised defensive repairingthat is, the healing processes.

On the other hand, the upper limit of the homeostatic window for SAMPs should not be exceeded because of the increased risk of hyperresolution associated with infectionpromoting immunosuppression, and the lower limit of the window should also not be crossed because of the increased risk of hyperinflammation and organ dysfunction or chronic inflammation. The final definition of such homeostatic 


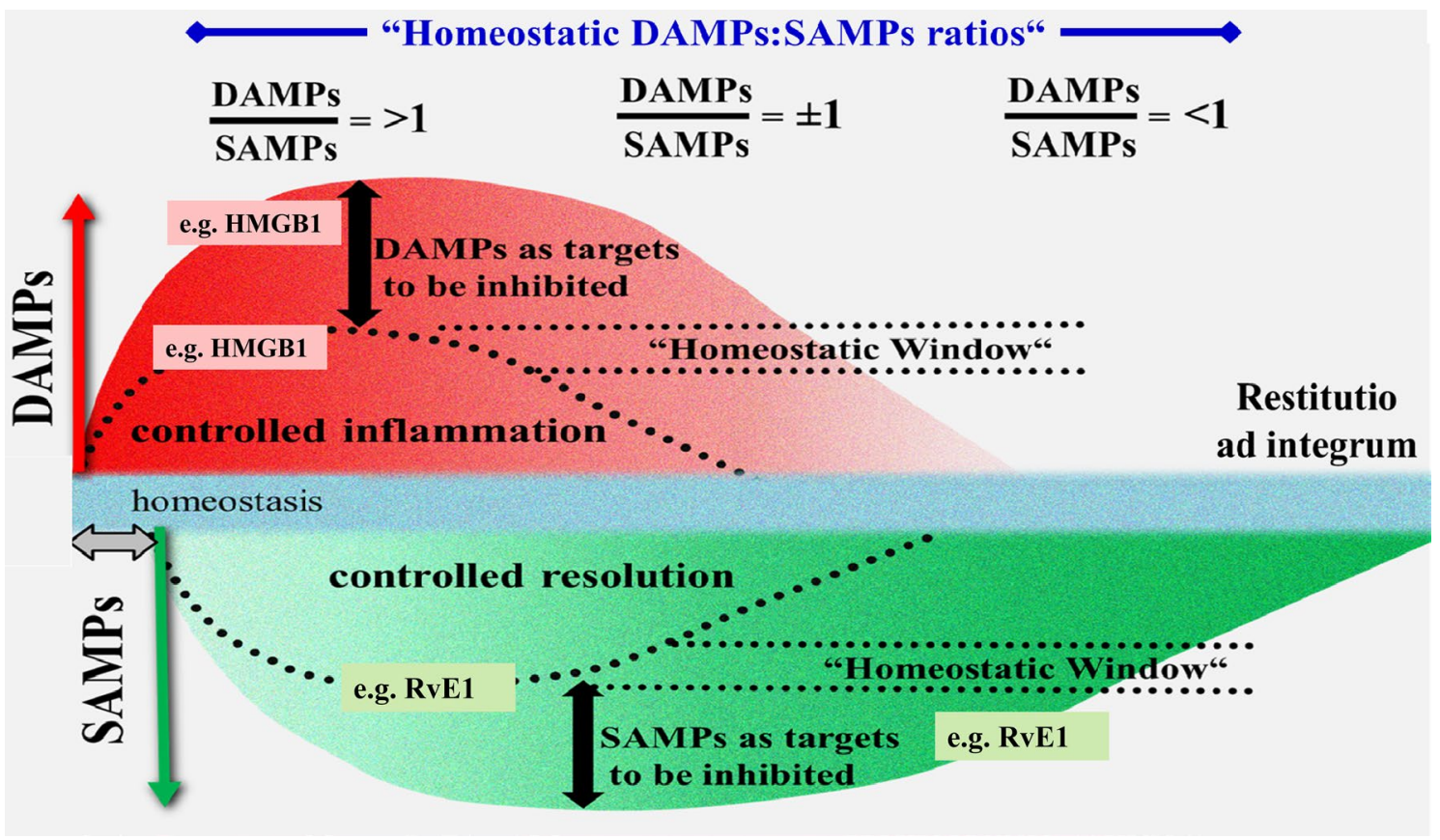

Fig. 1 Schematic diagram of the design of a narrative model illustrating a theoretical framework for a safe therapeutic strategy to inhibit excessive concentrations of damage-associated molecular patterns (DAMPs) and suppressing/inhibiting DAMPs (SAMPs) in hyperinflammation (e.g., systemic inflammatory response syndrome [SIRS]), followed by hyperresolution (e.g., compensatory anti-inflammatory response syndrome [CARS]). The core of such a model is the a priori elaboration of an approximately "homeostatic DAMP:SAMP ratio" by choosing the model of an injury-induced controlled inflammatory response as a reference point for the identification of such a ratio.

windows in adherence to the homeostatic DAMP:SAMP ratio may turn out to be a prerequisite for any therapeutic intervention modality to be chosen-although it is not an easy one to explore.

In addition, if an effective drug has been selected, for example, a blocking monoclonal antibody (mAb), the next step would be to define a therapeutic window for the drug's dosing, which would require careful pharmacokinetic, pharmacodynamic, and pharmacogenomic studies.

\subsubsection{Plasma Concentration of HMGB1, S100 Proteins, Circulating DNA, and Specialized Proresolving Mediators in Healthy Individuals (at a First Glance)}

Plausibly, as a first reference point for defining a homeostatic window for DAMPs and SAMPs, their concentrations as potential biomarkers should be known, as measured in healthy persons (i.e., baseline values) — clearly, still a blank spot on the map. Nevertheless, initial information is available about the concentrations of the prominent DAMPs HMGB 1, S100 proteins, and circulating DNA (i.e., nDNA
Based on (1) such an elaborated "homeostatic DAMP:SAMP ratio" and (2) targeted clinical monitoring of adverse events, a "homeostatic window" of DAMP and SAMP concentrations is recommended to be defined. The window of DAMP levels should not be exceeded because of the increased risk of hyperinflammation-associated multiple organ failure; the window of SAMP levels should not be exceeded because of the increased risk of immunosuppression-associated lifethreatening infections. $H M G B 1$ high mobility group box $1, R v E 1$ resolvin E1

and mtDNA), as found in healthy persons. Here, just for initial orientation, and not discussed in-depth, are a few examples.

For instance, healthy individuals reportedly express plasma HMGB1 levels with a mean of $1.65 \pm 0.04 \mathrm{ng} / \mathrm{mL}$, as assessed by standard enzyme-linked immunosorbent assay (ELISA) methods, and the isoform of this constitutive HMGB1 pool is the fully reduced and non-acetylated molecule. For assessment of post-translationally modified (hyperacetylated) HMGB1, liquid chromatography-mass spectrometry is currently the only technology available [43, 44] (to compare comments on this issue, see [15]; for the redox state of HMGB1, see [5], Vol. 1, Part IV, Sect. 12.2.2.5 and Fig. 12.2; for translationally modified secreted HMGB1, see [5], Vol. 1, Part IV, Sect. 14.3.3 and Fig. 14.4).

As for S100 proteins, their median plasma concentration in healthy volunteers has been estimated to be $0.052 \mu \mathrm{g} / \mathrm{L}$ [45]. In other studies, the median serum level of S100A4 was found to be $11.8(0-41.5) \mathrm{pg} / \mathrm{mL}$, which was significantly lower than the level in patients with acute myocardial infarction (MI), 89.6 (4.3-214.6) pg/mL. The cut-off value 
was set at $41.5 \mathrm{pg} / \mathrm{mL}$ (75th percentile of the S100A4 distribution in the controls) [46].

As for circulating DNA measured in healthy individuals, median nDNA and mtDNA plasma concentrations of $1.64 \times 103$ and $8.32 \times 107$ copies/mL (measured by quantitative polymerase chain reaction [Q-PCR] analysis technology), respectively, were found, corresponding to 5.43 and $1.36 \mathrm{ng} / \mathrm{mL}$ of plasma [47]. In other clinical studies in the context of investigations in patients with acute cardiac diseases, healthy individuals were found to have a plasma nDNA concentration (measured by Q-PCR) of $0.1683 \pm 0.0254 \mathrm{ng} / \mu \mathrm{L}$, and for mtDNA, a concentration of $0.1517 \pm 0.0924 \mathrm{ng} / \mu \mathrm{L}[48]$.

Plasma concentrations of SAMPs, here SPM profiles, have also been measured using liquid chromatographytandem mass spectrometry (LC-MS) technology [49, 50]. Of note, in a recently published evaluation of SPM profiles using an optimized LC-MS method for the detection of SPMs in biological samples, Kutzner et al. [51] concluded, "SPMs were generally not detectable/quantifiable in plasma and serum of healthy individuals, while good recovery rates were found in spiked samples. These results strongly support findings that circulating levels of SPMs are very low, i.e., $<0.1 \mathrm{nM}$ in healthy subjects. In samples from patients with end-stage renal disease (and peritonitis) or septic shock SPMs and precursors were detectable; however, not directly correlated with the health status and clinical outcome."

\subsubsection{Concluding Remarks}

It is not difficult to imagine that the workup of identifying and defining a context-dependent homeostatic DAMP:SAMP ratio and a homeostatic window of DAMP and SAMP concentrations to allow safe treatment options is a cumbersome clinical task. On the other hand, this situation is not new and has been previously encountered when a new kind of treatment targeting endogenous molecules was considered. Future clinical studies will have to show whether such expensive and extensive investigations are worthwhile and make sense.

\subsection{Caveats for Therapeutic Targeting of DAMPs and SAMPs in Hyperinflammation and Hyperresolution}

Blockade/inhibition of DAMPs in hyperinflammatory processes (e.g., systemic inflammatory response syndrome [SIRS]) should only be considered if the concentration exceeds the known "homeostatic window," in order not to jeopardize the proinflammatory protective defensive power of DAMPs. The same caveat has to be applied when considering the blockade/inhibition of SAMPs in potential hyperresolving processes. Here, it is crucial not to interfere with the resolving processes required for optimal homeostatic healing (see Fig. 1).

Probably the main challenge of such a novel therapeutic maneuver in hyperinflammation lies in choosing an optimal time period suitable for blocking the action of DAMPs on the one hand and subsequently inhibiting the action of SAMPs on the other. This procedure must be accurately dosed and finely tuned. Otherwise, for example, in the case of a too early blockade of DAMPs, potential pathogens may grow and spread out. On the other hand, too early inhibition of SAMPs may interfere with the beneficial repairing processes.

\subsection{DAMPs as Therapeutic Targets and SAMPs as Therapeutics in Chronic Inflammation: The DAMP:SAMP Ratio as the Guideline}

Today, chronic inflammation is seen as a state of non-resolving inflammation. Accordingly, two therapeutic options may be considered: inhibition of DAMPs as therapeutic targets and administration of SAMPs as therapeutics. Such maneuvers again imply that the ratio of DAMP:SAMP must be carefully measured at frequent intervals to allow proper tailor-made therapeutic decisions to be made.

For example, in the case where DAMPs are inhibited too much or SAMPs are administered in doses that are too large, over an excessive time period, regulatory T cells (Tregs) may be generated to promote immunosuppression [52-54], which is associated with increased susceptibility to infections.

In general, and when the pathogen in question is successfully controlled, as shown in the case of infection, a contextdependent combination of all three of the following concepts in moderate dosing might be the optimal therapeutic solution in chronic inflammation: (1) timely blockade of the action of DAMPs, (2) timely application/substitution of SAMPs, and (3) administration of anti-inflammatory agents.

\subsection{Résumé}

Harnessing DAMPs and SAMPs as therapeutic targets and therapeutics is an attractive novel therapeutic option, which will perhaps find its way into future routine treatment modalities for many human diseases. Nevertheless, one again has to realize that the intrinsic nature of DAMPs and SAMPs is to maintain and restore (potentially life-saving) homeostasis upon cell stress and tissue injury. A too drastic therapeutic manipulation of these molecules can result in dyshomeostasis, that is, pathologies and disorders. Physicians and clinicians will be confronted with a learning phase of how to handle such therapeutic interventions. Therefore, strict monitoring of the DAMPs and SAMPs within defined homeostatic windows during the whole course of the disease appears to be 
recommended. Some further aspects are mentioned in the following sections.

\section{DAMPs and SAMPs in Therapy: The Various Options in Practice}

\subsection{Introductory Remarks}

Besides the various therapeutic caveats regarding harnessing DAMPs and SAMPs as therapeutic targets and therapeutics, molecules need to be druggable, allowing the use of specific effective agents. This issue is briefly addressed in the following sections.

\subsection{DAMPs and SAMPs as Therapeutic Targets}

When choosing DAMPs as therapeutic targets, the DAMPs should be druggable molecules. Druggability is the property of a molecule (i.e., a biological target such as a protein or nucleic acid) that elicits a favorable clinical response when it contacts a drug or a drug-like compound. Drug targets should possess specific properties, including (1) binding with high affinity to a drug that must alter the function of the target, with a therapeutic benefit to the patient; (2) differential expression across the body for specific targeting; (3) the quality of a biomarker to monitor its efficacy; and (4) freedom to operate, that is, a lack of competitive binding [55-58]. Obviously, DAMPs fulfill these criteria.

The idea of choosing DAMPs, for example, HMGB1, as a therapeutic target to inhibit innate immune responses with the use of mAbs or biologicals has already been proposed by Land [59]. Today, several strategies are being discussed and applied to prevent release of DAMPs or to inhibit their activities. Such therapeutic maneuvers are worth considering in, for example, hyperinflammation (e.g., SIRS, as currently observed in Covid-19) and certain chronic inflammatory/ autoimmune diseases. Current pharmacological strategies include the use of mAbs, peptides, decoy receptors, and small molecules, but also absorption/adsorption procedures [60]. For example, plasma adsorption therapy was recently reported to adsorb HMGB1 efficiently [31]. In addition, as also discussed by Venereau et al. [61] and VanPatten and $\mathrm{Al}$-Abed [62], in the future, the development of specific HMGB1 inhibitors, such as specific small molecules, may potentiate and fine-tune the pharmacological control of inflammation.

Indications for which SAMPs may be chosen as therapeutic targets may be situations such as unwanted hyperresolution and/or immunosuppression, for example, those observed in compensatory anti-inflammatory response syndrome (CARS) following polytrauma [63]. Again, when choosing
SAMPs as therapeutic targets, they should be druggable molecules.

\subsection{SAMPs as Therapeutics in Chronic Inflammatory Processes}

The concept of impaired resolution leading to chronic inflammatory disorders, autoimmune diseases like rheumatoid arthritis, and Crohn's disease, as well as it contributing to allergic diseases such as asthma, has paved the way to consider SAMPs as therapeutics in activating inflammation-resolving processes. In principle, potential therapeutics include adenosine, AnxA1, and SPMs, but also SPM derivatives, other synthetic small molecule agonists of SPM receptors, inhibitors of SPM-degrading enzymes (e.g., soluble epoxide hydrolase inhibitors), and modifiers of the SPM synthesis pathways. With the indications mentioned, SPMs have already been proven to have potent proresolving actions in a range of disease models. Given their potency, many drug development opportunities are possible. As outlined by Serhan [64], aspirin and statins have a positive impact on these resolution pathways, producing epimeric forms of specific SPMs, whereas other drugs can disrupt timely resolution. As further outlined by this investigator [64], evidence from recent human and preclinical animal studies indicates that SPMs are physiological mediators and pharmacological agonists that stimulate resolution of inflammation and infection. This observation is reason enough to use them clinically as inhibitors and antagonists alone, and to develop immunoresolvents as agonists to test resolution pharmacology and their role in catabasis for their therapeutic potential [64]. Dalli [65] argues similarly regarding the potential of resolution pharmacology-based approaches in developing new therapeutics for combating infections that do not interfere with the immune response. This issue is also raised by Schett and Neurath [66], who discuss potential intervention strategies for fostering the resolution process and the implications for the therapy of inflammatory diseases.

Indeed, given the new concept of inflammation resolution as an essential part of inflammation biology and pathology, the development of SAMPs for clinical use is a dictate of the hour. As stated by Serhan in this context [67], "The available evidence from extensive preclinical animal models, human SPM production in vivo, and the limited results of randomized clinical trials in humans suggest that it is indeed time to consider stimulating resolution in the twentyfirst century as a new therapeutic direction for managing unwanted excessive inflammation and infection. Proresolution pharmacology can enhance the host innate response to expedite microbial clearance, limit collateral tissue damage, and stimulate tissue regeneration by enhancing endogenous resolution mechanisms that are programmed into the resolving exudates of the acute inflammatory response." 
Indeed, not only SPMs but also other SAMPs could stimulate the development of novel therapeutic strategies, encompassing a novel "resolution pharmacology" approach.

\subsection{DAMPs as Therapeutics to Boost Innate Resistance}

\subsubsection{General Remarks}

The intrinsic evolution-determined nature of DAMPs is to promote adaptive immune defense responses via activation of antigen-presenting dendritic cells (DCs) (reviewed in [5], Part VIII, Chpt. 32). This implies that they can be used as therapeutics in situations in which a robust immune defense response is desired. Typical examples refer to promotion of anti-pathogen-directed immune processes aimed at succeeding in strong vaccination effects and induction of immunogenic cell death (ICD) in cancer cells, sought to instigate a potent antitumor immune response.

\subsubsection{General Administration of DAMPs in Vaccination Procedures}

There is a wide indication field for the administration of DAMPs to boost short- and long-term innate resistance against pathogens and cancer cells. For a long time, adjuvants have been used in vaccine formulations to induce potent adaptive (humoral!) immune responses that cannot be achieved with antigen alone [68]. Aluminum hydroxide (alum) is the most common adjuvant currently in clinical use $[69,70]$ that has been denoted as an exogenous DAMP [5]. Of note, in studies in mice, alum was found to cause cell death associated with the subsequent release of host cell DNA identified by the authors as a DAMP [71]. However, one has to realize that-according to current knowledgecell death leads to release of large amounts of other DAMPs as well, which all mediate adjuvanticity. Thus, it is not a surprise that the ongoing development of novel vaccines intend to incorporate the combination of DAMP-inducing adjuvants and PAMP adjuvants [72].

\subsubsection{Induction of DAMPs in Antitumor Therapy}

The induction of ICD aimed at promoting a robust antitumor immune response is currently an emerging topic in oncology. Fired by the danger/injury model in immunology [1, 2, 73-76], a conceptual revolution in oncology has emerged in that cancer is considered to be entities that can be detected and destroyed by the immune system under certain circumstances. The core of this new concept refers to the phenomenon of ICD. Of note, however, only a few lethal stimuli are intrinsically endowed with the ability to instigate ICD, including certain chemotherapeutics, radiotherapy, certain oncolytic viruses, and photodynamic therapy [77]. They are called "ICD inducers" [78]. There is accumulating evidence indicating that these therapeutic inducers cause ICD through endoplasmic reticulum (ER) stress associated with or induced by reactive oxygen species (ROS) [79]. Typically, ICD is associated with the emission of a series of DAMPs that are generated in a precise spatiotemporally defined configuration. This coordinated emission of DAMPs then allows elicitation of a robust antitumor immune response that is associated with the establishment of immunological memory.

\subsubsection{Concluding Remarks}

The clinical use of DAMPs as therapeutics aimed at promoting adaptive immune responses is emerging, but is probably not restricted to vaccination and antitumor therapy. Other fields may be considered, for example, it may be imaginable to add certain DAMPs to antibiotic treatment in the case of intractable infections, such as that observed in infectious disorders caused by antibiotic-resistant pathogens.

\subsection{Résumé}

Here, in terms of a first design, some principles of the safe use of DAMPs and SAMPs as therapeutic targets and therapeutics have been tentatively introduced. Nevertheless, to implement these tasks clinically in the future, agonists to these molecules have to be developed, some are already available. For example, as reviewed by Venereau et al. [61] and Andersson et al. [15], there are reports from preclinical studies on the successful use of polyclonal or mouse/rat antiHMGB1 mAbs in a number of experimental inflammatory models. To enable development of HMGB1-targeted therapy for clinical use, humanized anti-HMGB1 mAbs have been designed, for example, a partly humanized, chimeric $\mathrm{mAb}$ targeting HMGB1 with preserved functionality compared to the parental mouse anti-HMGB1 mAb [80].

Thus, removing or neutralizing extracellular HMGB 1 in infectious or sterile inflammation would be a plausible approach to ameliorate the human diseases concerned. However, up to now, there has only been limited clinical evidence for therapeutic agents that target extracellular HMGB1, but several promising candidates are in preclinical or clinical development. As concluded by Andersson et al. [15], "Blocking excessive amounts of extracellular HMGB1, particularly the disulfide isoform, offers an attractive clinical opportunity to ameliorate systemic inflammatory diseases. Therapeutic interventions to regulate intracellular HMGB 1 biology must still await a deeper understanding of intracellular HMGB 1 functions. Future work is needed 
to create more robust assays to evaluate functional bioactivity of HMGB1 antagonists. Forthcoming clinical studies would also greatly benefit from a development of antibodybased assays to quantify HMGB1 redox isoforms, presently assessed by mass spectrometry methods.... Together, we believe that HMGB1-specific antagonists should be tested in multiple parallel clinical studies of inflammatory disease syndromes to reveal whether blocking extracellular HMGB1 will benefit patients."

Certainly, such opinions, statements, and conclusions must be discussed for antagonists of all the other critical DAMPs and SAMPs. As one can imagine, this research field-including the activities of the pharmaceutical industry-is currently in full swing, and many highly exciting reports can be expected in the near future, providing a promising expansion of the therapeutic spectrum to treat human diseases. Nevertheless, the crucial key question has not yet been answered satisfactorily: is it feasible to achieve a lasting therapeutic effect by inhibiting only one DAMP or SAMP, or do we always have to inhibit a cocktail of key DAMPs or SAMPs according to distinct determined DAMP or SAMP patterns?

\section{Open Questions and Unsolved Problems}

As partially touched on already, there is a lot of open questions and unsolved problems regarding the practical feasibility of the designed concept. In fact, this paper has been written to highlight these unresolved issues, rather than to present conclusive answers to them. For example, we cannot satisfactorily answer the question as to what distinct DAMPs and SAMPs, out of about $>35$ currently known subclasses of such molecules [5], are most significant to the calculation of the DAMP:SAMP ratio in a given disease. On the DAMP side, key molecules such as HMGB1, circulating free DNA, S100 proteins, and extracellular histones appear to be the first choice; on the SAMP side, the most studied SPMs are obviously the best candidates. Sophisticated clinical trials focused on specific diseases will have to be designed and conducted to gain sufficient knowledge regarding the key DAMPs and SAMPs involved in any given disease, allowing subsequent calculation of a ratio useful for therapeutic considerations.

For example, in trauma patients developing SIRS, the DAMP mtDNA was shown to reach plasma levels of $\sim 400 \mathrm{ng} / \mathrm{mL}$, compared to homeostatic mtDNA levels in healthy subjects of $\sim 4 \mathrm{ng} / \mathrm{mL}$ (for the exact data, see [81]). Taking $4 \mathrm{ng} / \mathrm{mL}$ as the homeostatic " 1 ," the DAMP increased by 100 in SIRS. On the other hand, as shown in other sets of studies on surviving sepsis patients, the plasma level of the SAMP LXA4 decreased to $~ 149 \mathrm{ng} / \mathrm{mL}$, compared to homeostatic LXA4 levels in controls of $\sim 277 \mathrm{ng} /$
$\mathrm{mL}$ (see [82]). Taking $277 \mathrm{ng} / \mathrm{mL}$ as the homeostatic "1," this SAMP decreased by 0.53 . According to such a tentatively constructed example, the DAMP:SAMP ratio for SIRS would be 100:0.53, that is, $>1$, increased by factor of +188 . As a therapeutic consequence, such data would be in favor of blocking this DAMP or substituting this SAMP, or even both. Certainly, such a calculation should be optimized by application of mathematical formulae, allowing the construction of a valid software-based algorithm for the exact timing and dosing of the selected agent.

Also, the usefulness of determining the DAMP:SAMP ratio during the complex pathogenetic interplay between DAMPs and SAMPs in a given disorder also has to be explored in future clinical trials. Such an interplay can be observed, for example, in healing after MI that is characterized by the sequelae (1) DAMP-controlled promotion of myocardial inflammation [83] $\rightarrow$ (2) SAMP-controlled resolution of inflammation [84] $\rightarrow$ (3) DAMP-promoted development of replacement fibrosis (reviewed in part in [85-92]). When these responses are dysregulated, for example, when DAMPs are emitted in excess or permanently, they may promote progressive reactive fibrosis, leading to late heart failure (for reviews, see [85-88, 93]). Consequently, DAMPs may be regarded as therapeutic targets to prevent MI-induced late heart failure [94].

The challenge for cardiologists here is not to interfere too early and aggressively with the DAMP-promoted inflammatory $\rightarrow$ fibrogenic/reparative responses, which induce healing replacement fibrosis. In other words, too early or too drastic inhibition of DAMP emission may lead to catastrophic cardiac rupture due to an insufficient DAMP-promoted repair response. On the other hand, inhibition of DAMP emission that is too late may lead to progressive profibrotic responses that may result in a reactive fibrosis associated with heart failure. Together, these therapeutically induced adverse effects might be avoided by meticulous monitoring of the DAMP:SAMP ratio, which would allow application of DAMP inhibitors at the right time and in the right dose.

Indeed, this and other issues not mentioned here have to be tackled and solved in future targeted clinical trials before the theoretical framework described above can be transformed into clinical reality.

\section{Outlook and Future Perspectives}

The topic addressed here opens new avenues for modern medicine. Introduction of DAMPs and SAMPs as biomarkers in everyday clinical life will certainly enrich the diagnostic and prognostic options of clinicians and may allow better risk stratification of life-threatening diseases.

Beyond these positive aspects, DAMPs as biomarkers should play an increasing role in future environmental 
research. Thus, strikingly, the environment has now been recognized to be the major contributor to the global disease burden $[95,96]$. Notably, $80-85 \%$ of human disease is reportedly linked to environmental exposures [97]. However, quantifying and specifying the myriad of environmental and lifestyle risk factors such as smoking, exposure to hazardous chemicals, and pathogenic microorganisms turned out to be very complex and difficult to manage [95, 98]. For example, persons can be exposed to a complex mixture of chemical and biological contaminants, with multiple sources, for varying durations across their life course.

To find a way out of this dilemma, the continuous endeavor of environmental research was intensified and culminated in the development of the "environmental health paradigm" [99]. This approach tries to integrate the knowledge of exposures and environmental health sciences by exploring the causal links between environmental exposure and adverse disease effects. Two prominent models emerged as a result of intense interdisciplinary discussions on the environmental health paradigm, called the exposome and the adverse outcome pathway concepts. The model of the environmental health paradigm-academically covered by the new field of exposure science [100]-has worldwide intensified efforts of modern environmental research to study the adverse effects of environmental agents on humans and animals. The main subject of such cross-disciplinary studies is the search for new tools to specify and assess the risk of adverse health outcomes caused by harmful environmental factors. Indeed, the field of environmental research is flourishing, as reflected by the establishment of an interdisciplinary area of environmental health sciences and public health.

Excitingly, there is accumulating evidence in support of the notion that both exogenous DAMPs (i.e., presented as harmful environmental factors) and endogenous DAMPs (i.e., caused by harmful environmental factors) represent an important integrating momentum of both concepts. Though not perfect, such an approach should represent a first step to integrate these unique molecules and their pathology-mediating functions into these two environmental health research paradigms. This momentum may give rise to an interesting tautological discussion on whether research on exogenous/endogenous DAMP-induced innate immune pathways and DAMP-shaped adaptive immune processes is just a specialised part of environmental health research or even the same topic! The final future aim of this approach certainly is to develop innovative DAMPbased in vitro tests for cumulative risk assessment, thereby allowing avoidance of the use of costly and (potentially) ethically flawed animal experiments.

Similarly, the introduction of DAMPs and SAMPs as therapeutic targets and therapeutics will certainly enrich the therapeutic options of clinicians and physicians and may allow new sophisticated and efficient treatment modalities indicated for all those disorders that are characterized by currently untreatable, dysregulated inflammatory responses, including inflammatory diseases caused by environmental exposure. In all these therapeutic efforts, however, it should not be forgotten that these molecules are responsible for the daily defense against any stress or injury. Also, here, the "Hippocratic oath" counts: "Primum non nocere."

\section{Compliance with Ethical Standards}

Funding No external funding was used in the preparation of this article.

Conflict of interest W.G. Land declares that he has no conflicts of interest that might be relevant to the contents of this article.

\section{References}

1. Land W, Schneeberger H, Schleibner S, Illner WD, Abendroth $\mathrm{D}$, Rutili G, et al. The beneficial effect of human recombinant superoxide dismutase on acute and chronic rejection events in recipients of cadaveric renal transplants. Transplantation. 1994;57:211-7.

2. Matzinger P. Tolerance, danger, and the extended family. Annu Rev Immunol. 1994;12:991-1045. https://www.ncbi.nlm.nih. gov/pubmed/8011301

3. Land W. Allograft injury mediated by reactive oxygen species: from conserved proteins of drosophila to acute and chronic rejection of human transplants. Part III: Interaction of (oxidative) stress-induced heat shock proteins with Toll-like receptor-bearing cells. Transplant Rev. 2003;17:67-86. https://linki nghub.elsevier.com/retrieve/pii/S0955470X0380006X

4. Heil M, Land WG. Danger signals-damaged-self recognition across the tree of life. Front Plant Sci. 2014;5:578. https:// www.ncbi.nlm.nih.gov/pubmed/25400647

5. Land WG. Damage-associated molecular patterns in human diseases; Vol. 1: Injury-induced innate immune responses. Springer International Publishing AG; 2018. https://www.sprin ger.com/de/book/9783319786544

6. Relja B, Land WG. Damage-associated molecular patterns in trauma. Eur. J. Trauma Emerg. Surg. 2019. https://www.ncbi. nlm.nih.gov/pubmed/31612270

7. Lamparello AJ, Namas RA, Constantine G, McKinley TO, Elster E, Vodovotz Y, et al. A conceptual time window-based model for the early stratification of trauma patients. J. Intern. Med. Blackwell Publishing Ltd; 2019, p. 2-15.

8. Rothammer A, Sage EK, Werner C, Combs SE, Multhoff G. Increased heat shock protein 70 (Hsp70) serum levels and low NK cell counts after radiotherapy - Potential markers for predicting breast cancer recurrence? Radiat Oncol. BioMed Central Ltd.; 2019; p 14.

9. Tajbakhsh A, Rezaee M, Barreto GE, Moallem SA, Henney NC, Sahebkar A. The role of nuclear factors as "Find-Me"/ alarmin signals and immunostimulation in defective efferocytosis and related disorders. Int. Immunopharmacol. Elsevier B.V.; 2020 . 
10. Dubois C, Marcé D, Faivre V, Lukaszewicz AC, Junot C, Fenaille F, et al. High plasma level of S100A8/S100A9 and S100A12 at admission indicates a higher risk of death in septic shock patients. Sci Rep. Nature Publishing Group; 2019; p. 9.

11. Dalli J, Colas RA, Quintana C, Barragan-Bradford D, Hurwitz S, Levy BD, et al. Human sepsis eicosanoid and proresolving lipid mediator temporal profiles. Crit Care Med. 2017;45:58-68. https ://www.ncbi.nlm.nih.gov/pubmed/27632672

12. Rosenberg JH, Rai V, Dilisio MF, Agrawal DK. Damage-associated molecular patterns in the pathogenesis of osteoarthritis: potentially novel therapeutic targets. Mol. Cell. Biochem. Springer New York LLC; 2017, p. 171-9.

13. Nanini HF, Bernardazzi C, Castro F, De Souza HSP. Damageassociated molecular patterns in inflammatory bowel disease: From biomarkers to therapeutic targets. World J Gastroenterol. 2018;2018:4622-34 (Baishideng Publishing Group Co., Limited).

14. Bresnick AR. S100 proteins as therapeutic targets. Biophys Rev. 2018;10:1617-29. https://www.ncbi.nlm.nih.gov/pubmed/30382 555.

15. Andersson U, Yang H, Harris H. Extracellular HMGB1 as a therapeutic target in inflammatory diseases. Expert Opin Ther Targets. 2018;22:263-77. https://www.ncbi.nlm.nih.gov/pubme $\mathrm{d} / 29447008$.

16. McIlroy DJ, Minahan K, Keely S, Lott N, Hansbro P, Smith DW, et al. Reduced deoxyribonuclease enzyme activity in response to high postinjury mitochondrial DNA concentration provides a therapeutic target for systemic inflammatory response syndrome. J Trauma Acute Care Surg. 2018;85:354-8. https://insights.ovid. com/crossref?an=01586154-201808000-00016.

17. Vijayakumar EC, Bhatt LK, Prabhavalkar KS. High Mobility Group Box-1 (HMGB1): a potential target in therapeutics. Curr Drug Targets. Bentham Science Publishers Ltd; 2019;20:1474-85.

18. Wang H, Anthony D, Selemidis S, Vlahos R, Bozinovski S. Resolving viral-induced secondary bacterial infection in COPD: A concise review. Front. Immunol. Frontiers Media SA.; 2018.

19. Lacerda JZ, Drewes CC, Mimura KKO, de Zanon CF, Ansari T, Gil CD, et al. Annexin A12-26 treatment improves skin heterologous transplantation by modulating inflammation and angiogenesis processes. Front Pharmacol. 2018;2018:9.

20. Ansari J, Kaur G, Gavins FNE. Therapeutic potential of annexin A1 in ischemia reperfusion injury. Int J Mol Sci. 2018;19(4). pii: E1211. https://doi.org/10.3390/ijms19041211.

21. Conte MS, Desai TA, Wu B, Schaller M, Werlin E. Pro-resolving lipid mediators in vascular disease. J Clin Invest Am Soc Clin Investig. 2018;2018:3727-35.

22. Recchiuti A, Mattoscio D, Isopi E. Roles, actions, and therapeutic potential of specialized pro-resolving lipid mediators for the treatment of inflammation in cystic fibrosis. Front Pharmacol. 2019;10:252. https://doi.org/10.3389/fphar.2019.00252.

23. Kooij G, Derada Troletti C, Leuti A, Norris PC, Riley I, Albanese $\mathrm{M}$, et al. Specialized pro-resolving lipid mediators are differentially altered in peripheral blood of patients with multiple sclerosis and attenuate monocyte and blood-brain barrier dysfunction. Haematologica. 2019; haematol.2019.219519. https://www. haematologica.org/lookup/doi/10.3324/haematol.2019.219519.

24. Brennan EP, Mohan M, Andrews D, Bose M, Kantharidis P. Specialized pro-resolving mediators in diabetes: novel therapeutic strategies. Clin Sci (Lond) NLM (Medline). 2019;133:2121-41.

25. Yin P, Wang X, Wang S, Wei Y, Feng J, Zhu M. Maresin 1 improves cognitive decline and ameliorates inflammation in a mouse model of Alzheimer's disease. Front Cell Neurosci. 2019;2019:13.
26. Kytikova O, Novgorodtseva T, Denisenko Y, Antonyuk M, Gvozdenko T. Pro-resolving lipid mediators in the pathophysiology of asthma. Med MDPI AG; 2019.

27. Machado MG, Tavares LP, Souza GVS, Queiroz-Junior CM, Ascenção FR, Lopes ME, et al. The annexin A1/FPR2 pathway controls the inflammatory response and bacterial dissemination in experimental pneumococcal pneumonia. FASEB J. 2019;fj.201902172R. https://onlinelibrary.wiley.com/doi/ abs/10.1096/fj.201902172R

28. Garg AD, Dudek AM, Agostinis P. Cancer immunogenicity, danger signals, and DAMPs: what, when, and how? Biofactors. 2013;39:355-67. https://www.ncbi.nlm.nih.gov/pubmed/23900 966

29. Land WG, Agostinis P, Gasser S, Garg AD, Linkermann A. DAMP-induced allograft and tumor rejection: the circle is closing. Am J Transplant. 2016;16:3322-37. https://www.ncbi.nlm. nih.gov/pubmed/27529775

30. Ahn J, Xia T, Rabasa Capote A, Betancourt D, Barber GN. Extrinsic phagocyte-dependent STING Signaling dictates the immunogenicity of dying cells. Cancer Cell. 2018;33:862-73.

31. Khodarev NN. Intracellular RNA sensing in mammalian cells: role in stress response and cancer therapies. Int Rev Cell Mol Biol. 2019;2019:31-89.

32. Wang G, Xu J, Zhao J, Yin W, Liu D, Chen WJ, et al. Arf1mediated lipid metabolism sustains cancer cells and its ablation induces anti-tumor immune responses in mice. Nat Commun Nature Res. 2020;2020:11.

33. Dalli J, Serhan CN. Specific lipid mediator signatures of human phagocytes: microparticles stimulate macrophage efferocytosis and pro-resolving mediators. Blood. 2012;120:e60-72. https:// www.ncbi.nlm.nih.gov/pubmed/22904297

34. Dalli J, Serhan C. Macrophage proresolving mediators-the when and where. Microbiol Spectr. 2016;4. https://www.ncbi.nlm.nih. gov/pubmed/27337457

35. Leuti A, Maccarrone M, Chiurchiù V. Proresolving lipid mediators: endogenous modulators of oxidative stress. Oxid Med Cell Longev. 2019;2019:1-12. https://www.ncbi.nlm.nih.gov/pubme $\mathrm{d} / 31316721$

36. Serhan CN, Levy BD. Resolvins in inflammation: emergence of the pro-resolving superfamily of mediators. J Clin Invest. 2018;128:2657-69. https://www.ncbi.nlm.nih.gov/pubme $\mathrm{d} / 29757195$

37. Li Y, Cai L, Wang H, Wu P, Gu W, Chen Y, et al. Pleiotropic regulation of macrophage polarization and tumorigenesis by formyl peptide receptor-2. Oncogene. 2011;30:3887-99. https ://www.nature.com/articles/onc2011112

38. Titos E, Rius B, González-Périz A, López-Vicario C, MoránSalvador E, Martínez-Clemente M, et al. Resolvin D1 and its precursor docosahexaenoic acid promote resolution of adipose tissue inflammation by eliciting macrophage polarization toward an M2-like phenotype. J Immunol. 2011;187:5408-18. https:// www.jimmunol.org/lookup/doi/10.4049/jimmunol.1100225

39. Dalli J, Zhu M, Vlasenko NA, Deng B, Haeggström JZ, Petasis NA, et al. The novel 13S,14S-epoxy-maresinis converted by human macrophages to maresin 1 (MaR1), inhibits leukotriene A4 hydrolase (LTA4H), and shifts macrophage phenotype. FASEB J. 2013;27:2573-83.

40. Rius B, Titos E, Morán-Salvador E, López-Vicario C, GarcíaAlonso V, González-Périz A, et al. Resolvin D1 primes the resolution process initiated by calorie restriction in obesity-induced steatohepatitis. FASEB J. 2014;28:836-48. https://www.ncbi. nlm.nih.gov/pubmed/24249635

41. Herová M, Schmid M, Gemperle C, Hersberger M. ChemR23, the receptor for chemerin and resolvin E1, is expressed and functional on M1 but not on M2 macrophages. J 
Immunol. 2015;194:2330-7. https://www.jimmunol.org/looku $\mathrm{p} /$ doi/10.4049/jimmunol.1402166

42. Wynn TA, Vannella KM. Macrophages in tissue repair, regeneration, and fibrosis. Immunity. 2016;44:450-62. https://www.ncbi. nlm.nih.gov/pubmed/26982353

43. Fukami A, Adachi H, Yamagishi S, Matsui T, Ueda S, Nakamura K, et al. Factors associated with serum high mobility group box 1 (HMGB1) levels in a general population. Metabolism. 2009;58:1688-93. https://linkinghub.elsevier.com/retrieve/pii/ S002604950900225X

44. Tang Y, Zhao X, Antoine D, Xiao X, Wang H, Andersson U, et al. Regulation of posttranslational modifications of HMGB1 during immune responses. Antioxid Redox Signal. 2016;24:62034. https://www.liebertpub.com/doi/10.1089/ars.2015.6409

45. Wiesmann M, Missler U, Gottmann D, Gehring S. Plasma $\mathrm{S}-100 \mathrm{~b}$ protein concentration in healthy adults is age- and sexindependent. Clin Chem. 1998;44:1056-8. https://www.ncbi. nlm.nih.gov/pubmed/9590385

46. Gong X-J, Song X-Y, Wei H, Wang J, Niu M. Serum S100A4 levels as a novel biomarker for detection of acute myocardial infarction. Eur Rev Med Pharmacol Sci. 2015;19:2221-5. https ://www.ncbi.nlm.nih.gov/pubmed/26166646

47. Meddeb R, Dache ZAA, Thezenas S, Otandault A, Tanos R, Pastor B, et al. Quantifying circulating cell-free DNA in humans. Sci Rep. 2019;9:5220. https://www.ncbi.nlm.nih.gov/pubmed/30914 716

48. Wang L, Xie L, Zhang Q, Cai X, Tang Y, Wang L, et al. Plasma nuclear and mitochondrial DNA levels in acute myocardial infarction patients. Coron Artery Dis. 2015;26:296-300. https ://www.ncbi.nlm.nih.gov/pubmed/25714070

49. Dalli J, Colas RA, Serhan CN. Novel n-3 immunoresolvents: structures and actions. Sci Rep. 2013;3:1940. https://www.ncbi. nlm.nih.gov/pubmed/23736886

50. Fosshaug LE, Colas RA, Anstensrud AK, Gregersen I, Nymo S, Sagen EL, et al. Early increase of specialized pro-resolving lipid mediators in patients with ST-elevation myocardial infarction. EBioMedicine. 2019;46:264-73. https://www.ncbi.nlm.nih.gov/ pubmed/31345784

51. Kutzner L, Rund KM, Ostermann AI, Hartung NM, Galano J-M, Balas L, et al. Development of an optimized LC-MS method for the detection of specialized pro-resolving mediators in biological samples. Front Pharmacol. 2019;10:169. https://www.ncbi.nlm. nih.gov/pubmed/30899221

52. Chiurchiù V, Leuti A, Dalli J, Jacobsson A, Battistini L, Maccarrone M, et al. Proresolving lipid mediators resolvin D1, resolvin D2, and maresin 1 are critical in modulating T cell responses. Sci Transl Med. 2016;8:353ra111. https://stm.sciencemag.org/looku p/doi/10.1126/scitranslmed.aaf7483

53. Krishnamoorthy N, Burkett PR, Dalli J, Abdulnour R-EE, Colas $\mathrm{R}$, Ramon $\mathrm{S}$, et al. Cutting edge: maresin- 1 engages regulatory $\mathrm{T}$ cells to limit type 2 innate lymphoid cell activation and promote resolution of lung inflammation. J Immunol. 2015;194:863-7. https://www.jimmunol.org/lookup/doi/10.4049/jimmunol.14025 34

54. Luo B, Han F, Xu K, Wang J, Liu Z, Shen Z, et al. Resolvin D1 programs inflammation resolution by increasing TGF- $\beta$ expression induced by dying cell clearance in experimental autoimmune neuritis. J Neurosci. 2016;36:9590-603. https://www.ncbi. nlm.nih.gov/pubmed/27629711

55. Imming P, Sinning C, Meyer A. Drugs, their targets and the nature and number of drug targets. Nat Rev Drug Discov. 2006;5:821-34. https://www.nature.com/articles/nrd2132

56. Davidson BL, McCray PB. Current prospects for RNA interference-based therapies. Nat Rev Genet. 2011;12:329-40. https:// www.nature.com/articles/nrg2968
57. Gashaw I, Ellinghaus P, Sommer A, Asadullah K. What makes a good drug target? Drug Discov Today. 2012;17:S24-30. https ://www.ncbi.nlm.nih.gov/pubmed/22155646

58. Zheng CJ, Han LY, Yap CW, Ji ZL, Cao ZW, Chen YZ. Therapeutic targets: progress of their exploration and investigation of their characteristics. Pharmacol Rev. 2006;58:259-79. https:// pharmrev.aspetjournals.org/cgi/doi/10.1124/pr.58.2.4

59. Land WG. Emerging role of innate immunity in organ transplantation part III: the quest for transplant tolerance via prevention of oxidative allograft injury and its consequences. Transplant Rev (Orlando). 2012;26:88-102. https://linkinghub.elsevier.com/retri eve/pii/S0955470X11000565

60. Gruda MC, Ruggeberg K-G, O'Sullivan P, Guliashvili T, Scheirer AR, Golobish TD, et al. Broad adsorption of sepsis-related PAMP and DAMP molecules, mycotoxins, and cytokines from whole blood using CytoSorb® sorbent porous polymer beads. Eller K, editor. PLoS One. 2018;13:e0191676. https://www.ncbi. nlm.nih.gov/pubmed/29370247

61. Venereau E, De Leo F, Mezzapelle R, Careccia G, Musco G, Bianchi ME. HMGB1 as biomarker and drug target. Pharmacol Res. 2016;111:534-44. https://linkinghub.elsevier.com/retri eve/pii/S104366181630487X

62. VanPatten S, Al-Abed Y. High mobility group box-1 (HMGb1): current wisdom and advancement as a potential drug target. J Med Chem. 2018;61:5093-107. https://www.ncbi.nlm.nih.gov/ pubmed/29268019

63. Sauaia A, Moore FA, Moore EE. Postinjury inflammation and organ dysfunction. Crit. Care Clin. 2017;2017:167-91.

64. Serhan CN. Treating inflammation and infection in the $21 \mathrm{st}$ century: new hints from decoding resolution mediators and mechanisms. FASEB J. 2017;31:1273-88. https://www.faseb j.org/doi/10.1096/fj.201601222R

65. Dalli J. Does promoting resolution instead of inhibiting inflammation represent the new paradigm in treating infections? Mol Aspects Med. 2017;58:12-20. https://www.ncbi.nlm.nih.gov/ pubmed/28365269

66. Schett G, Neurath MF. Resolution of chronic inflammatory disease: universal and tissue-specific concepts. Nat Commun. 2018;9:3261. https://www.ncbi.nlm.nih.gov/pubmed/30111884

67. Serhan CN. Treating inflammation and infection in the $21 \mathrm{st}$ century: new hints from decoding resolution mediators and mechanisms. FASEB J. 2017;31:1273-88. https://www.ncbi. nlm.nih.gov/pubmed/28087575

68. Desmet CJ, Ishii KJ. Nucleic acid sensing at the interface between innate and adaptive immunity in vaccination. Nat Rev Immunol. 2012;12:479-91. https://www.nature.com/articles/ nri3247

69. He P, Zou Y, Hu Z. Advances in aluminum hydroxide-based adjuvant research and its mechanism. Hum Vaccin Immunother. 2015;11:477-88. https://www.ncbi.nlm.nih.gov/pubme $\mathrm{d} / 25692535$

70. Powell BS, Andrianov AK, Fusco PC. Polyionic vaccine adjuvants: another look at aluminum salts and polyelectrolytes. Clin Exp Vaccine Res. 2015;4:23. https://www.ncbi.nlm.nih. gov/pubmed/25648619

71. Marichal T, Ohata K, Bedoret D, Mesnil C, Sabatel C, Kobiyama K, et al. DNA released from dying host cells mediates aluminum adjuvant activity. Nat Med. 2011;17:996-1002. https:// www.nature.com/articles/nm.2403

72. Hayashi T, Momota M, Kuroda E, Kusakabe T, Kobari S, Makisaka K, et al. DAMP-inducing adjuvant and PAMP adjuvants parallelly enhance protective type- 2 and type- 1 immune responses to influenza split vaccination. Front Immunol. 2018;9:2619. https://www.ncbi.nlm.nih.gov/pubmed/30515151

73. Land WG. Emerging role of innate immunity in organ transplantation part II: potential of damage-associated molecular 
patterns to generate immunostimulatory dendritic cells. Transplant Rev (Orlando). 2012;26:73-87. https://doi.org/10.1016/j. trre.2011.02.003.

74. Matzinger P. The evolution of the danger theory. Interview by Lauren Constable, Commissioning Editor. Expert Rev Clin Immunol. 2012;8:311-7. https://www.ncbi.nlm.nih.gov/pubme $\mathrm{d} / 22607177$

75. Land WG, Messmer K. The danger theory in view of the injury hypothesis: 20 years later. Front Immunol. 2012;3:349. https:// www.ncbi.nlm.nih.gov/pubmed/23189080

76. Land WG, Messmer K. The innate immune system: its rediscovery before toll was described. Innate Immun. Resist. Dis. Princ. Karger Publishers; 2013 p. 24-8. https://www.karger. com?doi=10.1159/000346531

77. Garg AD, Galluzzi L, Apetoh L, Baert T, Birge RBRB, BravoSan Pedro JMJM, et al. Molecular and translational classifications of DAMPs in immunogenic cell death. Front Immunol. 2015;6:588. https://www.ncbi.nlm.nih.gov/pubmed/26635802

78. Garg AD, Dudek-Peric AM, Romano E, Agostinis P. Immunogenic cell death. Int J Dev Biol. 2015;59:131-40. https://www. ncbi.nlm.nih.gov/pubmed/26374534

79. Garg AD, Krysko D V, Vandenabeele P, Agostinis P. The emergence of phox-ER stress induced immunogenic apoptosis. Oncoimmunology. 2012;1:786-8. https://www.ncbi.nlm.nih.gov/ pubmed/22934283

80. Lundbäck P, Lea JD, Sowinska A, Ottosson L, Fürst CM, Steen $\mathrm{J}$, et al. A novel high mobility group box 1 neutralizing chimeric antibody attenuates drug-induced liver injury and postinjury inflammation in mice. Hepatology. 2016;64:1699-710. https:// doi.wiley.com/10.1002/hep.28736

81. McIlroy DJ, Minahan K, Keely S, Lott N, Hansbro P, Smith DW, et al. Reduced deoxyribonuclease enzyme activity in response to high postinjury mitochondrial DNA concentration provides a therapeutic target for systemic inflammatory response syndrome. J Trauma Acute Care Surg. Lippincott Williams and Wilkins; 2018. p. 354-8. https://www.ncbi.nlm.nih.gov/pubmed/30080 781

82. Tsai W-H, Shih C-H, Yu Y-B, Hsu H-C. Plasma levels in sepsis patients of annexin A1, lipoxin A4, macrophage inflammatory protein-3a, and neutrophil gelatinase-associated lipocalin. J Chin Med Assoc. 2013;76:486-90. https://www.ncbi.nlm.nih.gov/ pubmed/23769883

83. Shah M, Yellon DM, Davidson SM. The Role of Extracellular DNA and Histones in Ischaemia-Reperfusion Injury of the Myocardium. Cardiovasc. Drugs Ther. Springer; 2020. https://www. ncbi.nlm.nih.gov/pubmed/32062794

84. Halade G V., Tourki B. Specialized pro-resolving mediators directs cardiac healing and repair with activation of inflammation and resolution program in heart failure. Adv Exp Med Biol. 2019. p. 45-64. https://www.ncbi.nlm.nih.gov/pubmed/31562 621

85. Frangogiannis NG. Cardiac fibrosis: Cell biological mechanisms, molecular pathways and therapeutic opportunities. Mol Aspects Med. 2019;65:70-99. https://linkinghub.elsevier.com/retrieve/ pii/S0098299718300670

86. Rischpler C. Acute myocardial infarction. Q J Nucl Med Mol Imaging. 2016;60:236-51. https://www.ncbi.nlm.nih.gov/pubme $\mathrm{d} / 27225319$
87. Prabhu SD, Frangogiannis NG. The biological basis for cardiac repair after myocardial infarction. Circ Res. 2016;119:91-112. https://www.ncbi.nlm.nih.gov/pubmed/27340270

88. Talman V, Ruskoaho H. Cardiac fibrosis in myocardial infarction-from repair and remodeling to regeneration. Cell Tissue Res. 2016;365:563-81. https://www.ncbi.nlm.nih.gov/pubme $\mathrm{d} / 27324127$

89. Krenning G, Zeisberg EM, Kalluri R. The origin of fibroblasts and mechanism of cardiac fibrosis. J Cell Physiol. 2010;225:6317. https://doi.wiley.com/10.1002/jcp.22322

90. Liaudet L, Rosenblatt-Velin N. Role of innate immunity in cardiac inflammation after myocardial infarction. Front Biosci (Schol Ed). 2013;5:86-104. https://www.ncbi.nlm.nih.gov/ pubmed/23277038

91. van den Borne SWM, Diez J, Blankesteijn WM, Verjans J, Hofstra L, Narula J. Myocardial remodeling after infarction: the role of myofibroblasts. Nat Rev Cardiol. 2010;7:30-7. https://www. nature.com/articles/nrcardio.2009.199

92. Shinde A V, Frangogiannis NG. Fibroblasts in myocardial infarction: a role in inflammation and repair. J Mol Cell Cardiol. 2014;70:74-82. https://linkinghub.elsevier.com/retrieve/pii/ S0022282813003477

93. Frangogiannis NG. Regulation of the Inflammatory Response in Cardiac Repair. Rosenzweig A, editor. Circ Res. 2012;110:15973. https://www.ncbi.nlm.nih.gov/pubmed/22223212

94. van Hout GP, Arslan F, Pasterkamp G, Hoefer IE. Targeting danger-associated molecular patterns after myocardial infarction. Expert Opin Ther Targets. 2016;20:223-39. https://www. ncbi.nlm.nih.gov/pubmed/26420647

95. Rappaport SM, Smith MT, Vandamme P, Brandt E De, Pot B, Foligné B, et al. Environment and Disease Risks. Science (80- ) 2010;330:460-1. https://www.ncbi.nlm.nih.gov/pubmed/20966 241

96. Manrai AK, Cui Y, Bushel PR, Hall M, Karakitsios S, Mattingly $\mathrm{CJ}$, et al. Informatics and data analytics to support exposomebased discovery for public health. Annu Rev Public Health. 2017;38:279-94. https://www.annualreviews.org/doi/10.1146/ annurev-publhealth-082516-012737

97. Uppal K, Walker DI, Liu K, Li S, Go Y-M, Jones DP. Computational metabolomics: a framework for the Million Metabolome. Chem Res Toxicol. 2016;29:1956-75. https://www.ncbi.nlm.nih. gov/pubmed/27629808

98. Willett WC. Balancing life-style and genomics research for disease prevention. Science. 2002;296:695-8. https://www.scien cemag.org/cgi/doi/10.1126/science. 1071055

99. Wilson SH, Schwartz DA. Disease-first: a new paradigm for environmental health science research. Environ Health Perspect. 2006;114:A398. https://www.ncbi.nlm.nih.gov/pubmed/16835 037

100. Lioy PJ, Smith KR. A discussion of exposure science in the 21 st century: a vision and a strategy. Environ Health Perspect. 2013;121:405-9. https://www.ncbi.nlm.nih.gov/pubmed/23380 895 